\title{
SISTEMA AUTOMÁTICO PARA DIAGNÓSTICO DE ÚLCERA DE CÓRNEA
}

\author{
AUTOMATIC SYSTEM FOR DIAGNOSTICS OF CORNEAL ULCER
}

Liliane Ventura ${ }^{1}$, Sidney Júlio de Faria e Sousa ${ }^{2}$, Caio Chiaradia ${ }^{3}$, Renato Coelho ${ }^{4}$ \& Mário Gazziro ${ }^{5}$

\begin{abstract}
${ }^{1}$ Coordenadora do Laboratório de Física Oftálmica, Pesquisadora; ${ }^{2}$ Docente, Diretor Clínico do Banco de Olhos do HCRP e Coordenador do Laboratório de Física Oftálmica; ${ }^{3}$ Aluno de graduação de Medicina - Faculdade de Medicina de Ribeirão Preto da Universidade de São Paulo. ${ }^{4}$ Aluno da pós-graduação de Física da Faculdade de Filosofia, Ciências e Letras de Ribeirão Preto da Universidade de São Paulo; ${ }^{5}$ Aluno de graduação em Engenharia de Produção da Universidade Federal de São Carlos.

CorRespondência: (Laboratório de Física Oftálmica) - Departamento de Oftalmologia da Faculdade de Medicina de Ribeirão Preto Campus Universitário - CEP:14048-900 - Ribeirão Preto - SP - FAX: (016) 633-0186. e-mails: liliane@ifqsc.sc.usp.br
\end{abstract}

VENTURA L et al. Sistema automático para diagnóstico de úlcera de córnea. Medicina, Ribeirão Preto, 30: 379-382, jul./set. 1997.

RESUMO: Úlceras corneanas ${ }^{1}$ são feridas na córnea, com alta potencialidade de cegueira, pois, mesmo curadas, tendem a tirar a transparência. Nos países em desenvolvimento e de natureza agrícola, elas respondem por cerca de $5 \%$ das causas de cegueira. Uma das formas de 0 clínico acompanhar o tratamento delas é pela estimativa subjetiva de sua dimensão. Evoluções favoráveis tendem a promover uma diminuição da ferida. No entanto, as medidas existentes para essa estimativa são rudimentares. Assim, estamos desenvolvendo um sistema ótico, automático, para a avaliação da área afetada, a ser implementado num hospital público (400 pacientes são analisados por semana no Setor de Doenças Externas Oculares). O sistema ótico é implementado numa Lâmpada de Fenda e acoplado a um detector do tipo CCD. A imagem é apresentada num monitor de vídeo para PC, através de uma placa de interface comercial, e está sendo desenvolvido um software dedicado à determinação da área de úlcera de córnea.

UNITERMOS: Úlcera da Córnea. Doenças da Córnea.

\section{1 - INTRODUÇÃO}

Para uma melhor compreensão, uma breve introdução a respeito da córnea humana será feita a seguir.

\section{A Córnea Humana ${ }^{2}$}

A parte anterior do olho humano, de aspecto transparente, é a córnea. Ela possui, de fora para dentro, as seguintes camadas: o epitélio, o estroma e o endotélio (veja a Figura 1).

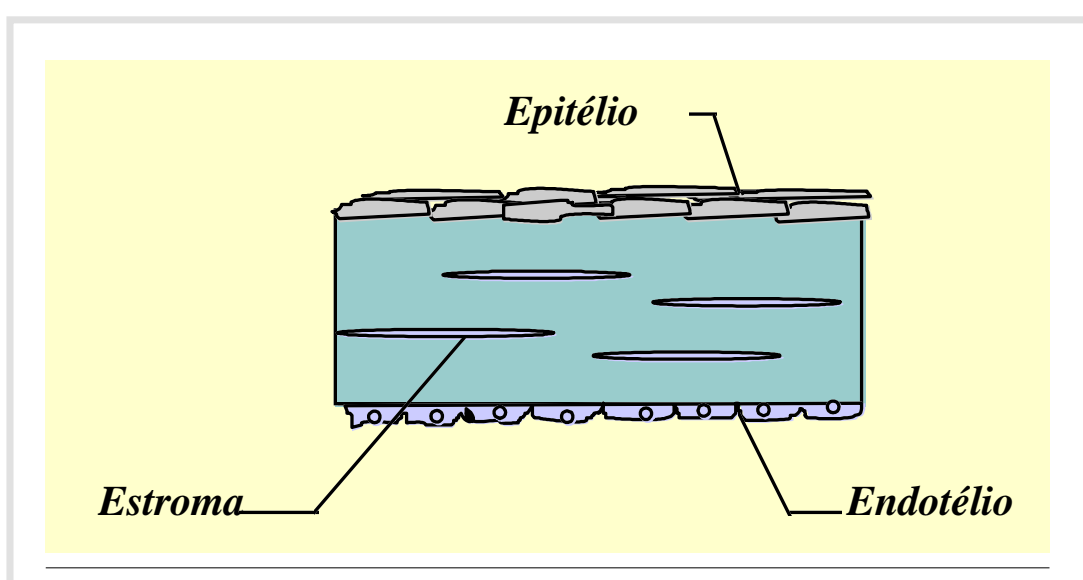

Figura 1 - Esquematização da córnea humana. 
O epitélio é o revestimento externo da córnea. Funciona como uma proteção mecânica, da mesma forma como a pele proteje as demais regiões de nosso organismo.

O estroma é a córnea propriamente dita, pois corresponde a 95\% da espessura da mesma. É constituído por tecido fibroso, disposto em lamelas regulares, com hidratação muito baixa e sem nenhum vaso sangüíneo. Tais características respondem por sua transparência acentuada.

Finalmente, o endotélio reveste a face interna da córnea com uma camada única de células poligonais.

\section{Úlcera de Córnea}

Algumas condições agridem o epitélio corneano, provocando solução de continuidade na mesma. Se, nessa situação, algum germe acesta-se no estroma desnudo, teremos configurada uma úlcera de córnea de origem infecciosa. A úlcera (Figura 2) é uma enfermidade particularmente grave, pelo alto potencial de cegueira. Acontece que, mesmo quando curada, tende a deixar uma zona de opacificação suficientemente importante para dificultar a visão. Na verdade, nos países agrícolas, de baixo nível sócio-econômico, essa enfermidade tende a responder por cerca de 1 a $5 \%$ das causas de cegueira uniocular (dados obtidos das estatísticas do HCRP-USP).

$\mathrm{Na}$ avaliação da resposta terapêutica das úlceras, entra em jogo uma série de variáveis clínicas, como o grau de infiltração de células inflamatórias no estroma, grau de hidratação da lesão, profundidade e extensão da ulceração. Todas essas variáveis são poten-

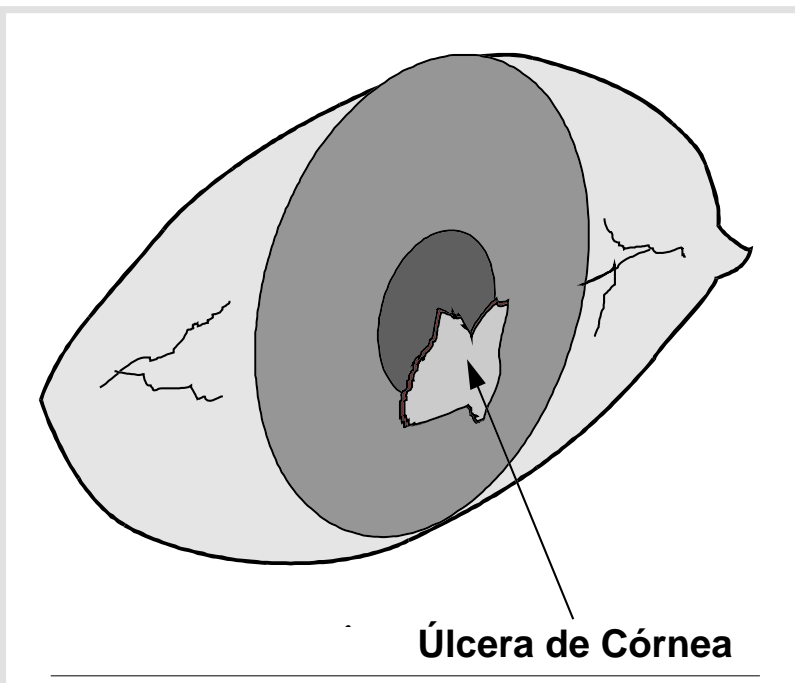

Figura 2 - Esquematização da úlcera corneana. cialmente difíceis de serem mensuradas, exceto a extensão da ulceração. Na verdade, um dos parâmetros mais utilizados pelo clínico, na identificação de uma evolução favorável, é a diminuição da área da úlcera. Acontece que mesmo essa estimativa é altamente subjetiva, por falta de instrumento adequado de medida. Quando muito, o médico mede a extensão horizontal da úlcera e infere sobre sua área. Outras vezes, compara-a com a área de iluminação de seu biomicroscópio para criar sua impressão. Os processos mais sofisticados incluem a fotografia da área da úlcera, através da qual, após a revelação do filme, pode-se melhor estimar aquela área; contudo, isso não ocorre instantaneamente. Assim, faz-se necessária uma medida automatizada da área da úlcera.

Portanto, este trabalho consiste no desenvolvimento de um sistema ótico para ser acoplado a uma Lâmpada de Fenda (instrumento utilizado para analisar as córneas) que, através de um detector do tipo $\mathrm{CCD}$ (charge coupled device - fotodetectores como os utilizados em câmeras de vídeo) e uma placa de interface CCD-PC, enviará as imagens ao microcomputador. Um "software" dedicado e interativo para o delineamento e cálculo da área ulcerada foi desenvolvido, bem como um banco de dados (ainda sofrendo alterações para sua melhoria).

Este sistema já está sendo implantado no ambulatório do Hospital das Clínicas da Faculdade de Medicina de Ribeirão Preto - USP e uma estatística do aprimoramento da qualidade do diagnóstico será feita.

\section{2 - MATERIAIS E MÉTODOS}

O paciente é submetido ao procedimento clínico usual para a visualização da úlcera, isto é, gotas de fuoresceína são instiladas no olho, por um clínico, e o paciente é posicionado à Lâmpada de Fenda. A Lâmpada de Fenda proporciona, através de um filtro para luz branca, uma luz azul que incide no olho, e a úlcera fluoresce no comprimento de onda verde (Figura 3).

O método experimental utilizado consiste em capturar a imagem da úlcera (imagem de 600X800 pixels, em 256 cores) a partir da Lâmpada de Fenda. Um sistema ótico, composto por duas lentes, uma íris e um prisma reto, são acoplados ao carona da Lâmpada de Fenda e a um detector CCD (Hitachi KP-550), que, por sua vez, está acoplado a uma placa de vídeo comercial (SE-100; tempo real). 
A imagem é capturada com boa resolução (570 linhas de TV) e é tratada pelo software desenvolvido e que determina a área da úlcera de córnea.

\section{3 - RESULTADOS}

O processamento da imagem é feito numa seqüencia de eventos. Primeiramente, os pixels pertencentes à úlcera são selecionados. Há duas sub-rotinas: uma delas seleciona os pixels pertencentes à úlcera, levando em conta a cor verde, e a outra seleciona os pixels, considerando sua intensidade. As Figuras 4a e 4b mostram a apresentação do software e o contorno de uma úlcera com a determinação de sua área, respectivamente.

O segundo passo consiste em usar um filtro, no intuito de se evitarem ruídos, e de contar o número de pixels incluídos na área de interesse. O número de pixels é convertido em $\mathrm{mm}^{2} \mathrm{e}$ a área é determinada com alta precisão $\left(0,01 \mathrm{~mm}^{2}\right)$.

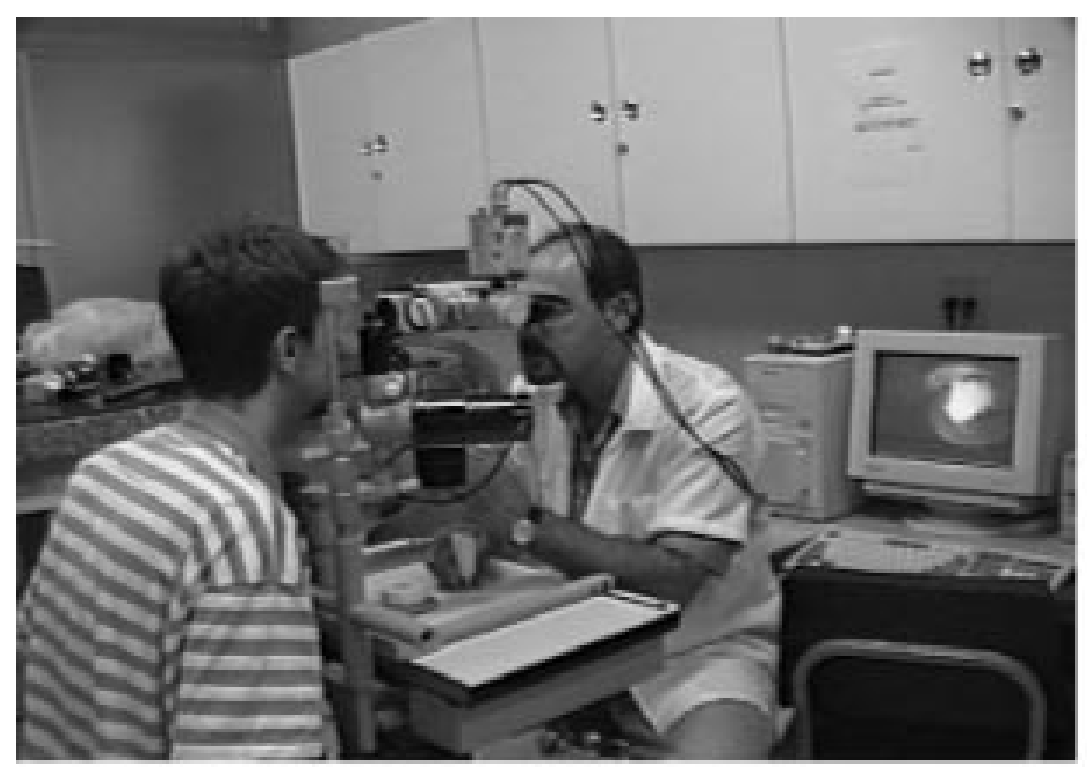

Figura 3 - Paciente submetido a uma avaliação de úlcera corneana.

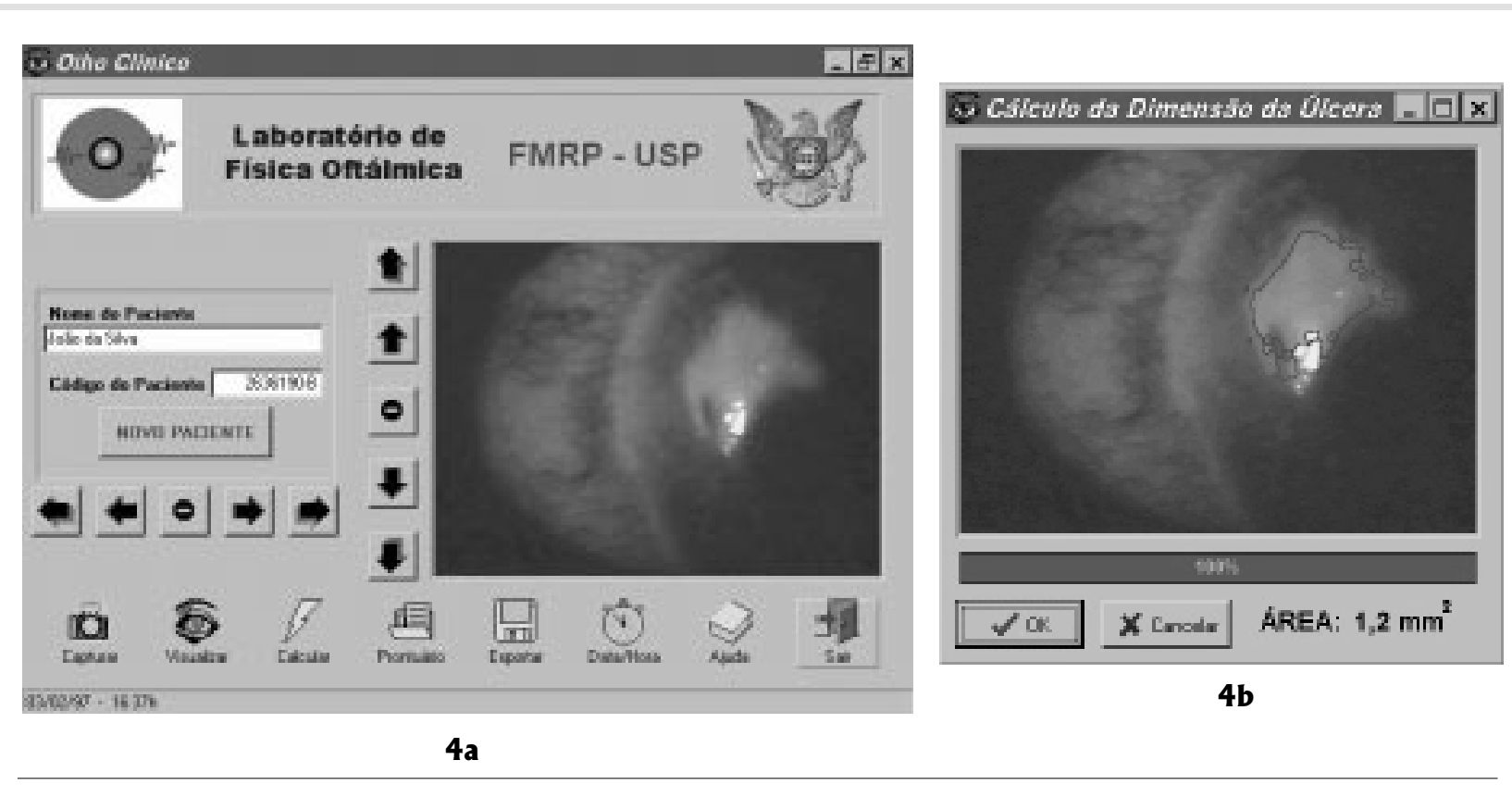

Figura 4 - A apresentação do software (4a); O contorno da úlcera de córnea (4b). 


\section{4 - DISCUSSÕES E CONCLUSÕES}

O sistema está em vias de se tornar um protótipo. Tem apresentado resultados satisfatórios, considerando a boa qualidade de imagem e a precisão obtida na determinação da área de úlcera corneana. No entanto, o tempo de processamento ainda é longo (40s para um microcomputador Pentium $166 \mathrm{MHz}$, 32 MB RAM) e deverá ser diminuído através do aprimoramento da técnica de processar a imagem, nos próximos meses.

O custo do sistema é comparável ao do sistema tradicional que envolve o processo fotográfico, porém, se o clínico já dispuser de um microcomputador e de uma câmera de vídeo normalmente utilizados em Lâmpada de Fenda para outras finalidades, o custo é extremamente baixo (praticamente o custo do adaptador ótico e do software).

O banco de dados já foi desenvolvido, e o programa é de fácil uso para o clínico (apresentação em ambiente Windows).
O sistema está sendo implementado no ambulatório do HCRP para que, nos próximos meses, seja utilizado como equipamento de rotina do Hospital. Desta forma, a evolução na avaliação e diagnóstico de úlceras de córnea poderá ser determinada, bem como uma estatística dos benefícios do sistema poderá ser feita posteriormente.

\section{5 - AGRADECIMENTOS}

Os autores gostariam de agradecer à FAPESP pelo suporte financeiro em relação a equipamentos, materiais de consumo e bolsas de estudo; ao CNPq e à CAPES pelas bolsas de estudo; ao RHAE, à FFCLRP-USP, ao IFSC-USP, ao Departamento de Oftalmologia da FMRP-USP pelo suporte estrutural e financeiro ao Laboratório de Física Oftálmica e ao Setor de Doenças Oculares Externas do HCRP-USP pela imensa colaboração para os testes, durante o desenvolvimento.

VENTURA L et al. Automatic system for diagnostics of corneal ulcer. Medicina, Ribeirão Preto, 30: 379-382, july/sept. 1997.

ABSTRACT: Corneal ulcer $^{1}$ is a very common disease in agricultural countries and it is responsible for $5 \%$ of the blindness causes. One of the main aspects to be observed in these cases is the increasing or decreasing of the affected area. Favourable evolutions decrease the wound. However the procedure of measuring these ulcers area are still very rudimentary.

We have been developing an automatic optical system in order to evaluate the affected area (the ulcer) to be implemented in a public hospital (400 patients per week are analyzed at the External Ocular Deseases sector). The optical system is implemented in a Slit Lamp and connected to a CCD detector. The image is displayed in a PC video monitor by a commercial frame grabber and a dedicated software for determining the area of the ulcer has been developed.

UNITERMS: Corneal Ulcer. Corneal Diseases.

\section{REFERÊNCIAS BIBLIOGRÁFICAS}

1 - UPADHYAY MP et al. Epidemiologic characteristics, predisposing factors and etiologic diagnosis of corneal ulceration in Nepal Am J Ophthalmol 111: 92-99, 1991.
2 - KRACHEMER JH; MANNIS MJ \& HOLLAND EJ. The corneal ulcer. In: Cornea: Fundamentals of cornea and external disease. Mosby, St. Louis, v. 1, cap. 25, p. 403-408, 1997.

Recebido para publicação em 11/03/97

Aprovado para publicação em 30/07/97 